\title{
Blood Coagulation Simulations using a Viscoelastic Model
}

\author{
A. Sequeira ${ }^{*}$, T. Bodnár ${ }^{2}$ \\ ${ }^{1}$ Department of Mathematics and CEMAT/IST, Instituto Superior Técnico, University of Lisbon \\ Av. Rovisco Pais 1, 1049-001 Lisboa, Portugal \\ ${ }^{2}$ Faculty of Mechanical Engineering, Czech Technical University in Prague \\ Karlovo Náměstí 13, 12135 Prague 2, Czech Republic
}

\begin{abstract}
This paper presents numerical results based on a macroscopic blood coagulation model coupled with a non-linear viscoelastic model for blood flow. The system of governing equations is solved using a central finite-volume scheme for space discretization and an explicit Runge-Kutta time-integration. An artificial compressibility method is used to resolve pressure and a non-linear TVD filter is applied for stabilization. A simple test case of flowing blood over a clotting surface in a straight $3 \mathrm{D}$ vessel is solved. This work presents a significant extension of the previous studies [10] and [9].
\end{abstract}

Keywords and phrases: blood coagulation, clot, viscoelastic model, numerical simulations

Mathematics Subject Classification: 76Z05, 76V05, 80A32, 92C35, 74F25, 80A30, 92C45, 92C40

\section{Introduction}

Mathematical and numerical modeling of blood flow and related phenomena are very challenging problems. The flow of blood is difficult to model mainly due to its complex rheological behavior which can be highly non-Newtonian in certain regimes in healthy and unhealthy blood flow conditions $[17,29,30]$. Rheological characteristics like shear-thinning viscosity, thixotropy, viscoelasticity or yield stress can be observed. A variety of models have been developed for blood flow including some of these features and, nowadays, it is still not possible to accept a single model to capture the most important mechanical properties of blood. The modeling approach is usually based on the blood flow conditions in view of predicting its behavior. The work presented here follows previous studies [11-13] describing the shear-thinning and viscoelastic behavior of blood in simple geometries. Even if blood flow is considered as purely mechanical, its mathematical modeling and numerical simulations present serious challenges as shown e.g. in $[18,21]$ or $[26]$.

The complex mechanical properties of blood flow are highly affected by the behavior of blood cellular elements (red blood cells, white blood cells and platelets), in particular by the aggregation of red blood cells into 3D microstructures at low shear rates and their disaggregation at high shear rates. Moreover,

${ }^{*}$ Corresponding author. E-mail: adelia.sequeira@math.ist.utl.pt

(C) EDP Sciences, 2014 
in flowing blood an enormous amount of biochemical reactions are associated to the complex process of blood coagulation and hemostasis.

Blood coagulation is an extremely complex biological process following a wound or a spontaneous internal injury, in which blood forms clots to prevent bleeding. The process involves platelets activation, the subsequent release of multiple factors from their cytoplasmatic granules, their interaction with the endothelial wall, with a huge impact of the flowing blood and on the thrombus growth regularization. The clot formation is followed by its dissolution repairing the injured wall, which is due to mechanical factors such as high shear stress (see e.g. $[16,28]$ ). The process of platelets activation and blood coagulation is not yet well understood. Numerous experimental studies have been performed and recent reviews detailing this complex process are available in the literature (as in e.g. [16] and references cited therein). A large number of sound phenomenological models for clot formation and lysis, based on different strategies, have been developed $[6,14,25,33]$ or $[1,24]$. One of the major problems is the high complexity of the chemical system which crucially depends on the supply of chemicals by the flowing blood. This leads to an important dependence of the coagulation process on the blood flow. On the other hand the blood flow characteristics are determined by the size of the clot, forming an obstacle to the flow.

Any reliable model of blood coagulation should integrate a large number of biochemical, physiologic and rheological factors and be validated by experimental data. The aim of this paper is to present a successful way of coupling two of the most complex macroscopic continuum based models of blood flow and biochemistry proposed in $[1,3]$. The work presented herein merges and significantly extends our previous studies [10] and [9]. We refer the reader to those papers for details concerning the model development and implementation, since they are essential to fully understand the present work.

The main added features introduced in this new paper are related to the extension of the coupled coagulation model presented in [10] by switching from the simple generalized Newtonian model to a new non-linear viscoelastic model that was previously implemented and tested in [9]. In comparison with our previous papers, the clot evolution is simulated on a longer time-interval, giving a better picture of the long-term behavior of the clotting process. In addition, the mechanical effects of the clot formation on the perturbation of the surrounding blood flow is studied in more detail than in our previous works.

\section{Mathematical Models}

The mathematical model consists of a coupled system of nonlinear partial differential equations describing the flow of blood and a biochemical cascade of reactions leading to the clot formation.

\subsection{Blood Flow Model}

The flow is described by a nonlinear shear-thinning and viscoelastic fluid model following the thermodynamics framework established in [27] and extended for blood flow in [4]. The set of governing equations is based on the conservation of mass (reduced to a divergence-free constraint) and the conservation of linear momentum for an incompressible fluid

$$
\begin{aligned}
\nabla \cdot \boldsymbol{u} & =0 \\
\rho\left(\frac{\partial \boldsymbol{u}}{\partial t}+\boldsymbol{u} \cdot \nabla \boldsymbol{u}\right) & =\nabla \cdot \boldsymbol{T} .
\end{aligned}
$$

The stress tensor $\boldsymbol{T}$ is split as follows:

$$
\boldsymbol{T}=-p \mathbf{1}+\mu \boldsymbol{B}_{\kappa_{p}(t)}+\eta_{1} \boldsymbol{D}
$$

where $\boldsymbol{D}=\left(\boldsymbol{L}+\boldsymbol{L}^{T}\right) / 2$ denotes the symmetric part of the velocity gradient tensor $\boldsymbol{L}, \mathbf{1}$ stands for the identity tensor and $\mu, \eta_{1}$ are positive material parameters. 
The upper-convected time-derivative ${ }^{1}$ of the elastic stretch tensor $\boldsymbol{B}_{\kappa_{p}(t)}$ is given by ${ }^{2}$ :

$$
\stackrel{\nabla}{\boldsymbol{B}}_{\kappa_{p(t)}}=-\frac{1}{\tau\left(\boldsymbol{B}_{\kappa_{p}(t)}\right)}\left[\boldsymbol{B}_{\kappa_{p}(t)}-\lambda \boldsymbol{1}\right]
$$

Here the $\tau=\tau\left(\boldsymbol{B}_{\kappa_{p}(t)}\right)$ defined by equation (2.6) has the dimension of time and plays a role similar to the relaxation time in the classical Oldroyd-B (Maxwell) model [11].

$$
\frac{1}{\tau\left(\boldsymbol{B}_{\kappa_{p}(t)}\right)}=2 K\left(\operatorname{tr}\left(\boldsymbol{B}_{\kappa_{p}(t)}\right)-3 \lambda\right)^{n}
$$

The coefficient $\lambda$ depends on the trace of the inverse of the tensor $\boldsymbol{B}_{\kappa_{p}(t)}$ according to

$$
\lambda=\frac{3}{\operatorname{tr}\left(\boldsymbol{B}_{\kappa_{p(t)}^{-1}}^{-1}\right.} \quad .
$$

The remaining model coefficients for blood are taken from [4]:

$$
\eta_{1}=0.01 \mathrm{~Pa} \cdot s ; \quad \mu=0.1611 \mathrm{~N} / \mathrm{m}^{2} ; \quad n=0.5859 ; \quad K=\left(\frac{\mu}{\alpha}\right)^{1+2 n}=58.0725 \mathrm{~s}^{-1}
$$

Using the definition of the upper-convected time derivative (2.4), the left-hand side can be rewritten in a more conventional form in terms of the material time-derivative:

$$
\frac{d \boldsymbol{B}_{\kappa_{p}(t)}}{d t}-\left[\boldsymbol{L} \boldsymbol{B}_{\kappa_{p}(t)}+\boldsymbol{B}_{\kappa_{p}(t)} \boldsymbol{L}^{T}\right]=-\frac{1}{\tau\left(\boldsymbol{B}_{\kappa_{p}(t)}\right)}\left[\boldsymbol{B}_{\kappa_{p}(t)}-\lambda \boldsymbol{1}\right]
$$

Finally, expanding the material time-derivative on the left-hand side we end up with

$$
\frac{\partial \boldsymbol{B}_{\kappa_{p}(t)}}{\partial t}+(\boldsymbol{u} \cdot \boldsymbol{\nabla}) \boldsymbol{B}_{\kappa_{p}(t)}=-\frac{1}{\tau}\left[\boldsymbol{B}_{\kappa_{p}(t)}-\lambda \boldsymbol{1}\right]+\left[\boldsymbol{L} \boldsymbol{B}_{\kappa_{p}(t)}+\boldsymbol{B}_{\kappa_{p}(t)} \boldsymbol{L}^{T}\right]
$$

where the coefficients $\lambda$ and $\tau$ are scalar functions of the tensor $\boldsymbol{B}_{\kappa_{p}(t)}$ and its invariants. More details related to this model and its implementation can be found e.g. in [9].

\subsection{Biochemistry Model}

The biochemistry model is based on a coupled set of non-linear advection-diffusion-reaction (ADR) equations. It has been originally developed in [1] and further extended in [3]. It describes the spatio-temporal evolution of concentrations $\left[C_{i}\right]$ of 23 chemical constituents (enzymes, zymogens, proteins, etc.) that can be written as

$$
\frac{\partial\left[C_{i}\right]}{\partial t}+\boldsymbol{u} \cdot \boldsymbol{\nabla}\left[C_{i}\right]=\boldsymbol{\nabla} \cdot\left(D_{i} \boldsymbol{\nabla}\left[C_{i}\right]\right)+R_{i}
$$

The non-linear chemical reaction terms $R_{i}$ are mainly based on first order, second order or MichaelisMenten kinetics. As an example we mention the reaction term $R_{I a}$ in the equation for fibrin production ${ }^{3}$ :

$$
R_{I a}=\frac{k_{1}[I I a][I]}{K_{1 M}+[I]}-\frac{h_{1}[P L A][I a]}{H_{1 M}+[I a]}
$$

\footnotetext{
${ }^{1}$ The upper-convected Oldroyd derivative of a tensor $\boldsymbol{A}$ is defined by

$$
\stackrel{\nabla}{\boldsymbol{A}}=: \frac{d \boldsymbol{A}}{d t}-\boldsymbol{L} \boldsymbol{A}-\boldsymbol{A} \boldsymbol{L}^{T}
$$

${ }^{2}$ The subscript $\kappa_{p}(t)$ is used to emphasize that the stretch is expressed with respect to the natural (time dependent) configuration $\kappa_{p}(t)$. This notation has been used in [4,27] and [9] where the model has been introduced and used.

${ }^{3}$ The subscript $I a$ refers to the chemical notation for factor Ia, an activated protein named fibrin.
} 
The concentrations of thrombin (denoted by $[I I a]$ ), fibrinogen (denoted by $[I]$ ), fibrin (denoted by $[I a]$ ) and plasminogen (denoted by $[P L A]$ ) are used to evaluate the reaction term $R_{I a}$. The chemical kinetics rates $k_{1}, h_{1}$ and the constants $K_{1 M}, H_{1 M}$ are known in the literature (taken from [3]). The values of the diffusion parameters $D_{i}$ and the exact form of the reaction terms $R_{i}$ are given in [10], where the model has been implemented for the first time and used in $3 \mathrm{D}$ simulations.

\subsection{Coupling Strategy}

The clot is a gel like structure consisting of a polymer (fibrin network), entrapping various blood components [16] and thus fibrin concentration can be used as an indicator of the clot formation. The main idea is to make the material properties of blood/clot dependent on fibrin concentration. For low fibrin concentration the fluid behaves like flowing blood, while for high fibrin concentration the fluid changes its behavior to a clot-like medium. In our model the fluid viscosity is multiplied by a factor $\tilde{\eta}_{1}$ that locally depends (linearly, up to a certain saturation value $\eta^{*}$ ) on the fibrin concentration $[I a]$. The viscosity $\eta_{1}$ is multiplied by a non-dimensional factor $\tilde{\eta}_{1}$

$$
\tilde{\eta}_{1}=\min \left\{1+\frac{\eta^{*}-1}{C_{c l o t}}[I a], \eta^{*}\right\}
$$

In our study we have used $\eta^{*}=100$ and $C_{\text {clot }}=1000 \mathrm{nM}$.

In this framework the clot is characterized as a highly viscous fluid. The region occupied by the simulated clot represents an obstacle to the flow field, with much lower viscosity. This effect is even significantly magnified due to the non-Newtonian shear-thinning behavior of blood, leading to a further increase of the fluid viscosity in regions of low shear.

Changes in the viscosity modify the local flow field which consequently affects the concentration field that leads to further changes in viscosity. In this way the interaction biochemistry-flow through this coupling strategy is enforced. More details can be found in [10] where this technique has been used for a generalized Newtonian model, i.e. neglecting the viscoelasticity. In the present study the technique has been extended to the new non-linear viscoelastic model as suggested in $[2,3,5]$.

\section{Numerical Methods}

The system of governing equations is rather complex and highly non-linear. This is why the numerical discretization has been chosen to be as simple and predictable as possible. We do not claim this choice is optimal, it only serves as the first step allowing the evaluation of the underlying mathematical model and to test its applicability in simple configurations. A semi-discretization approach is adopted to first discretize the PDEs in space and then integrating the resulting system of ODEs in time, both for the blood flow and the biochemical model.

The space discretization is based on a simple central finite-volume discretization on a structured grid with hexahedral cells. A multiblock grid topology with wall-fitted cells is used. The viscous fluxes are also discretized using a finite-volume method over a diamond-shaped cells adjoint to primary control volumes faces. This approach was used in our previous papers $[8,11,13]$ or in $[22,23]$.

The time integration is performed using a Runge-Kutta (RK) multistage scheme. A specific advectiondiffusion optimized RK method is used to reduce the computational cost. The basic idea behind this subclass of RK methods is to split the space-discretization operator into its inviscid and viscous parts. The inviscid part is evaluated in every stage of the RK method while the viscous fluxes are only evaluated in a few stages. This corresponds to an operator splitting technique with different RK methods (coefficients) used for the advection step and another for the diffusion step. This allows to save several (very expensive) evaluations of diffusive fluxes per time-step while retaining the rather large stability region of the RK method. For details see $[19,20]$ or $[11,13]$.

Along with these two basic components of the numerical solver a specific stabilization technique is used to avoid non-physical numerical oscillations due to the central discretization. The non-linear TVD filter $[15,32]$ was used to smooth the concentration fields, as recently reported in [7]. 


\section{Numerical Results}

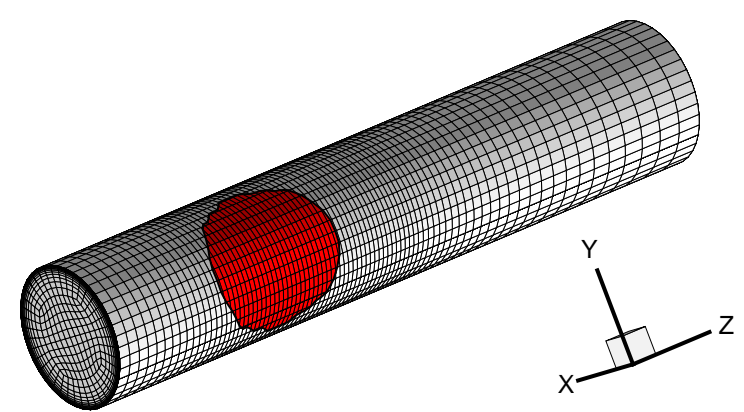

FIGURE 1. Grid structure and clotting surface position.

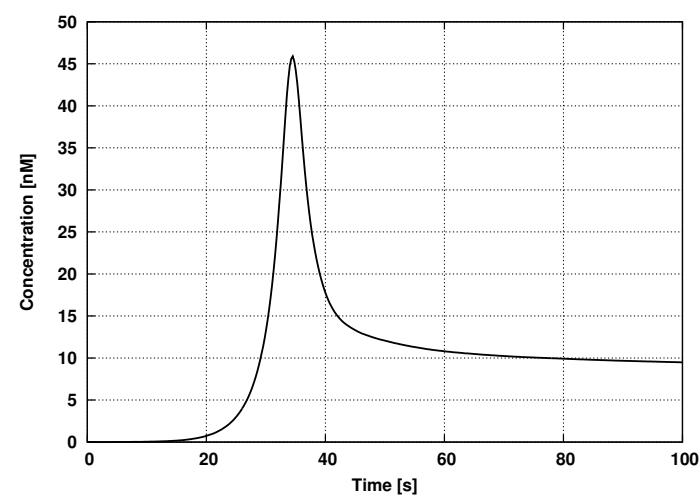

(a) Thrombin

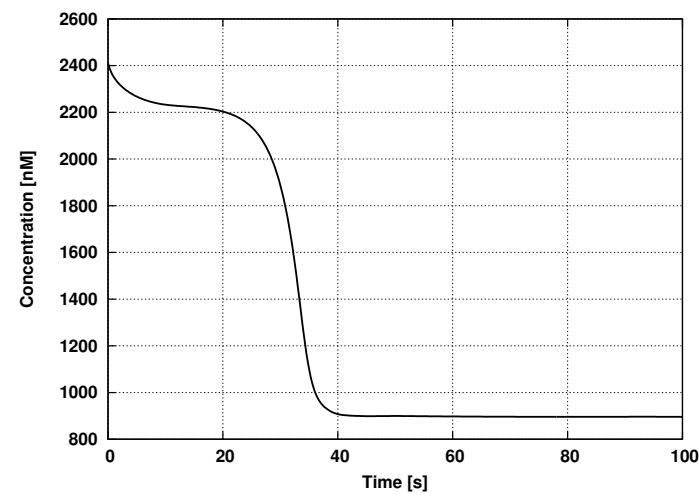

(c) Anti-Thrombin III

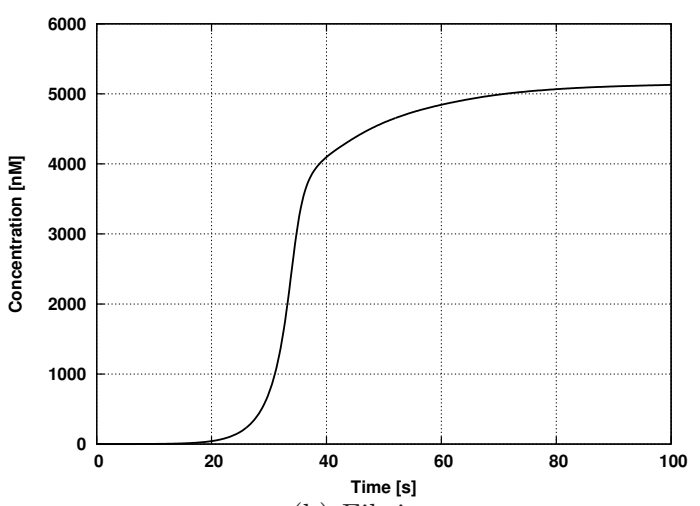

(b) Fibrin

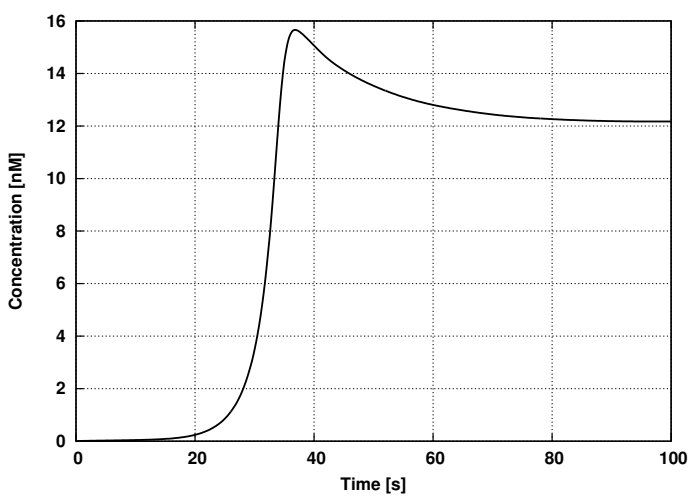

(d) Tissue Plasminogen Activator

Figure 2. Time evolution of selected concentrations in the center of the clotting surface during the initial 100 seconds. 


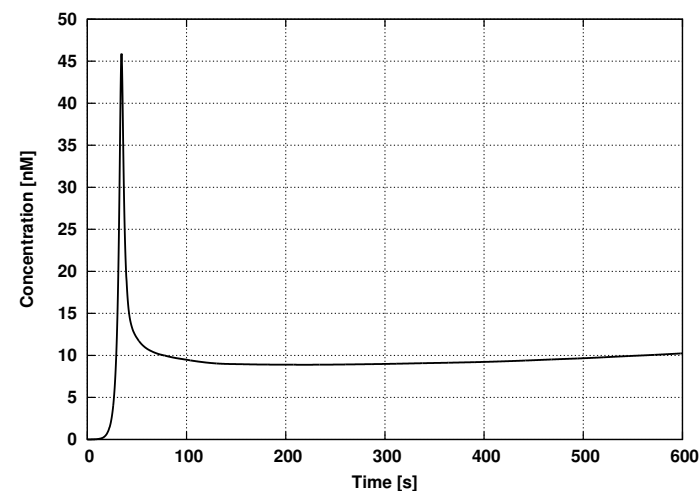

(a) Thrombin

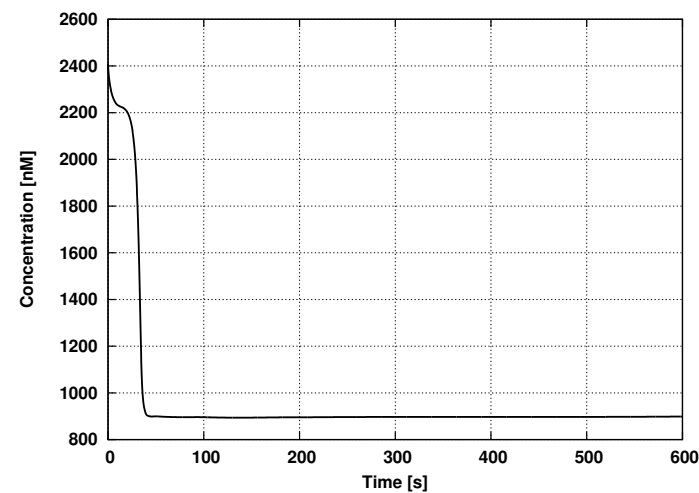

(c) Anti-Thrombin III

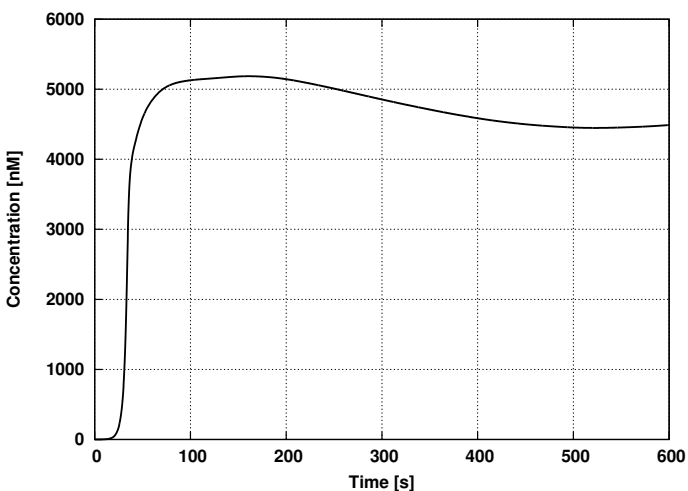

(b) Fibrin

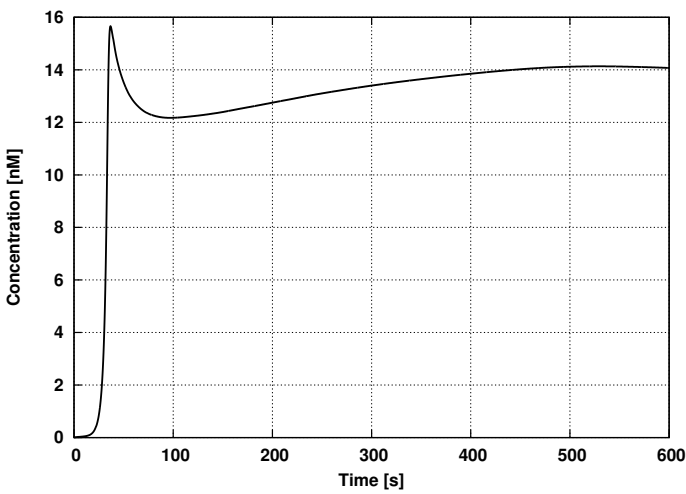

(d) Tissue Plasminogen Activator

FIgURE 3. Time evolution of selected concentrations in the center of the clotting surface during the initial 10 minutes.

The numerical test case is similar to the one used in [10] and [9], where we refer the complete parameter set for this simulation. The geometry represents a straight section of a blood vessel with diameter $6.2 \mathrm{~mm}$ and length $31 \mathrm{~mm}$ with grid shown in Figure 1.

The clotting surface is simulated in a region that is formed by the intersection of a sphere (of radius $3.1 \mathrm{~mm}$ ) with the blood vessel wall. The evolution in space and time of the clot is tracked down.

The model is very complex and thus the simulations generate large amounts of data to be visualized, analyzed, and understood. In this paper only a few snapshots of results are presented to demonstrate some of the most important model outputs.

The evolution in time of some of the coagulation factors can be observed in figures 2 and 3 . The concentration is visualized in a single point located in the center of the clotting surface on the vessel wall. Only the initial 600 seconds of clotting are shown.

These graphs show the nature of the coagulation process, initially very fast, with rather slow long term evolution in the later phase.

The spatial extent of the clot can be shown using the fibrin concentration contours. Figure 4 shows those contours on the surface of the blood vessel.

The velocity field is affected by the clotting which results in flow deceleration and velocity magnitude reduction in the regions of high fibrin concentration. To quantify and visualize the local flow deceleration, 


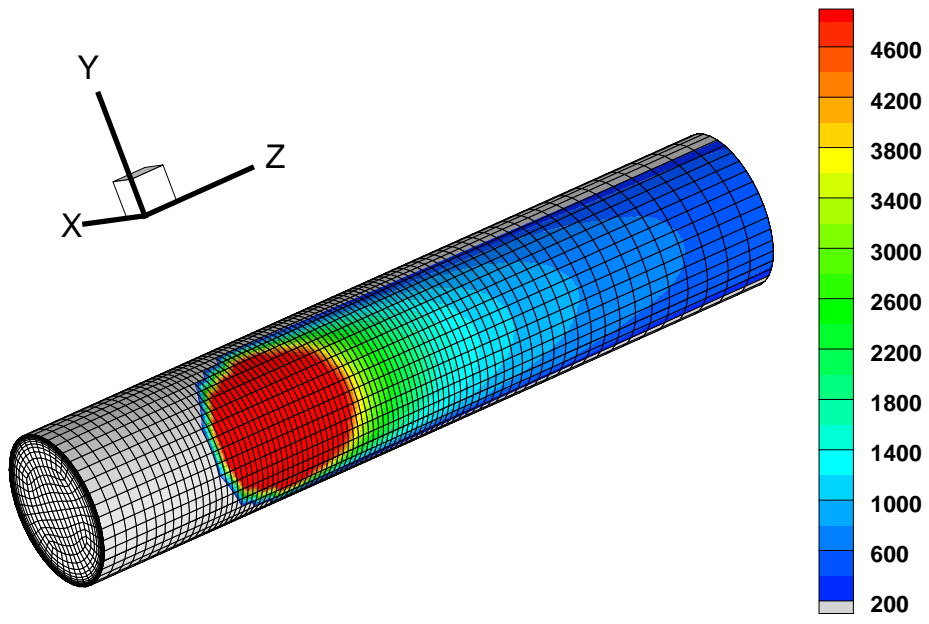

FIGURE 4. Fibrin concentration contours on the vessel wall at time $100 \mathrm{~s}$.

the non-dimensional velocity reduction $\delta$ is defined as

$$
\delta(\boldsymbol{x}, t)=\frac{q(\boldsymbol{x}, 0)-q(\boldsymbol{x}, t)}{q(\boldsymbol{x}, 0)} .
$$

Here $q(\boldsymbol{x}, t)$ is the local velocity magnitude $q=\sqrt{u^{2}+v^{2}+w^{2}}$. Since the no-slip condition is imposed on the vessel wall, the velocity reduction is evaluated in the first near-wall grid node.

A snapshot of flow velocity magnitude reduction $\delta$ is shown in Figure 5.

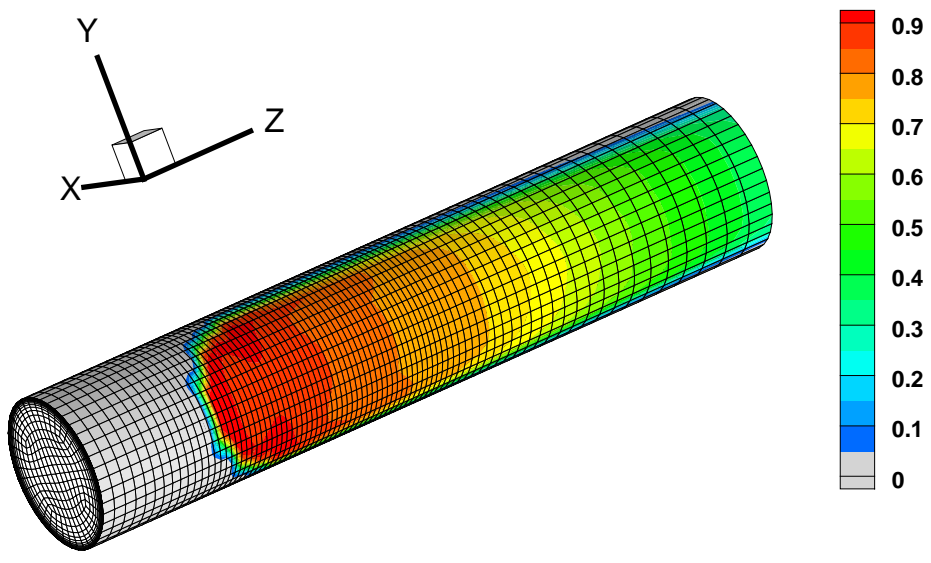

FiguRE 5. Near wall velocity magnitude reduction $\delta$ at time $100 \mathrm{~s}$. 
Time evolution of the clot growth and its effects on blood flow can be observed in Figure 6 showing the fibrin concentration and the velocity reduction $\delta$ on the deployed vessel surface ${ }^{4}$.
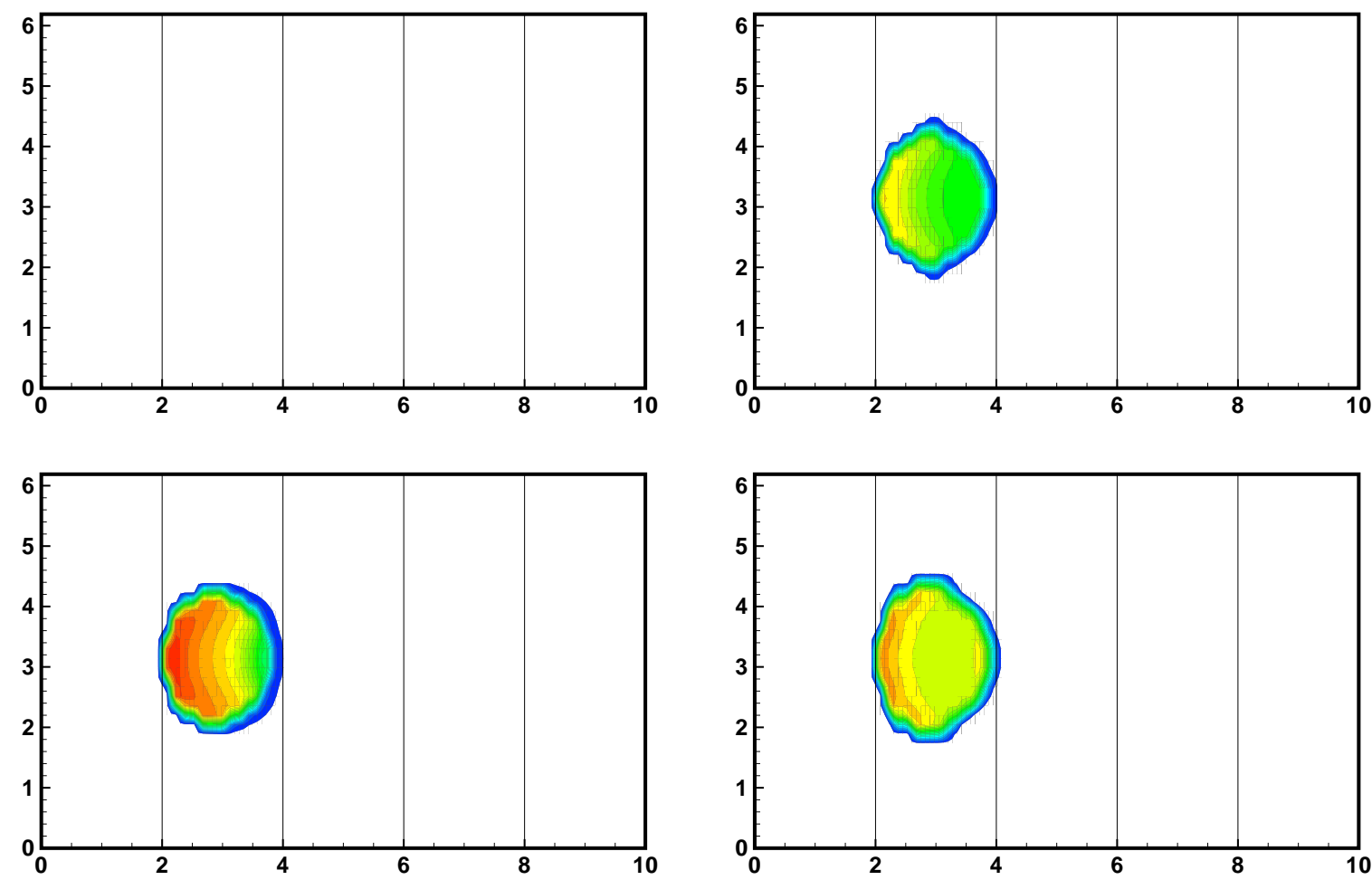

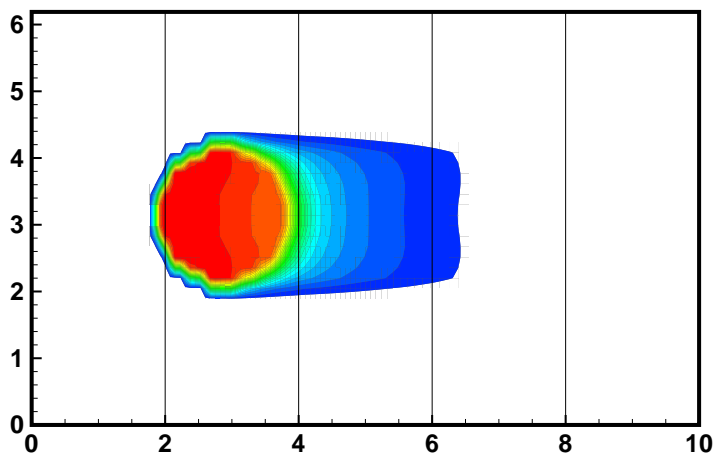

(a) Fibrin concentration $[I a]$

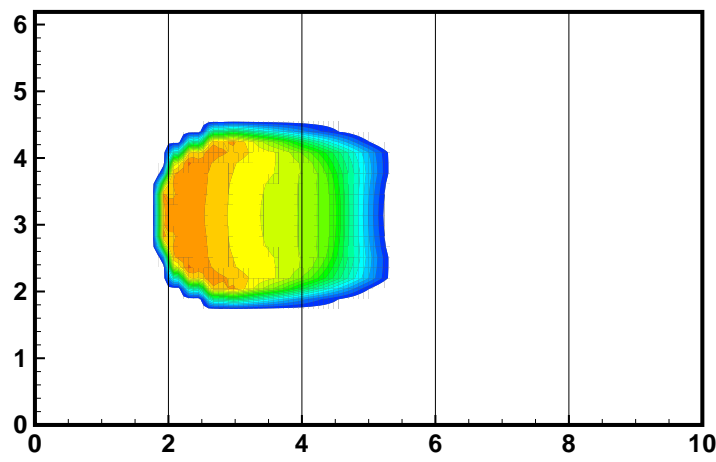

(b) Blood velocity reduction $\delta$

Figure 6. Clot evolution during the initial 60 seconds: snapshots at times $t=$ $20,40,60 s$, from top to bottom.

\footnotetext{
${ }^{4}$ The spatial dimensions are normalized using the vessel radius (half-diameter) $R=3.1 \mathrm{~mm}$.
} 
The same kind of visualization, but for a longer period of 600 seconds was used in Figure 7 .
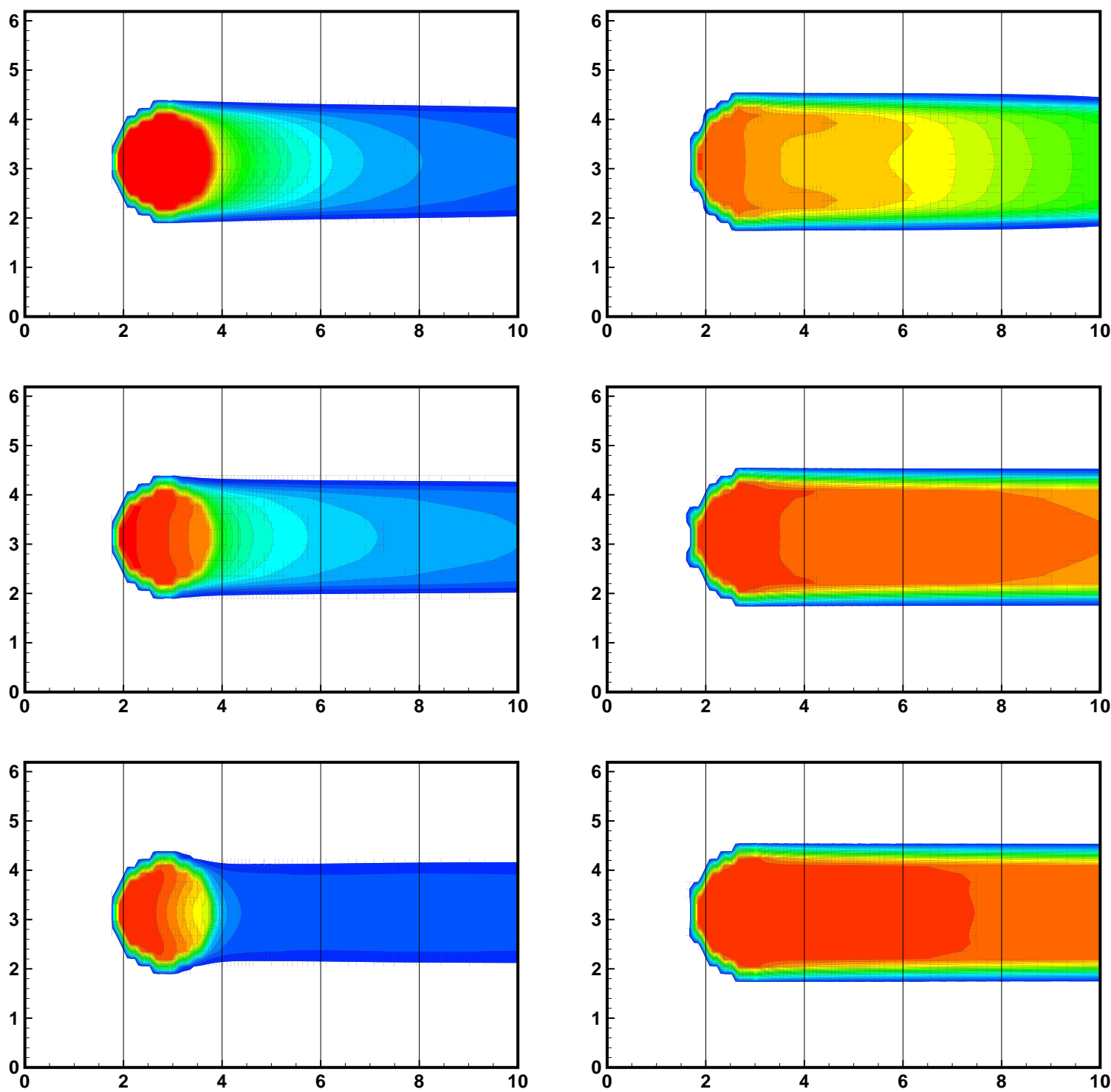

(a) Fibrin concentration $[\mathrm{Ia}]$

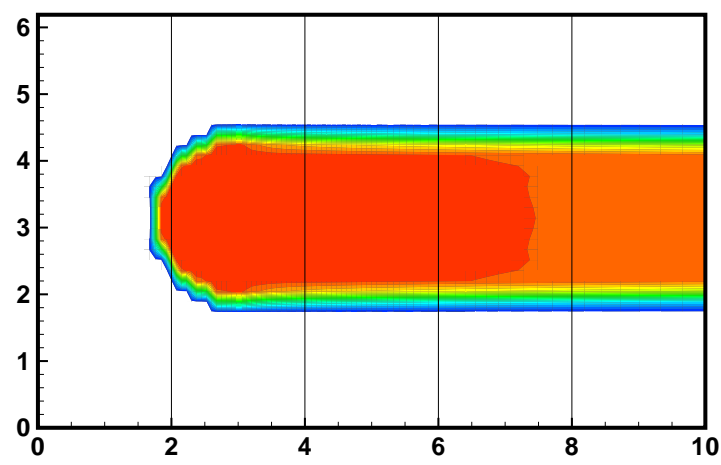

(b) Blood velocity reduction $\delta$

Figure 7. Clot evolution during the initial 600 seconds: snapshots at times $t=$ $120,360,600 s$, from top to bottom.

A better picture of the fibrin levels during the initial phase of clotting can be seen in Figure 8 showing the concentrations along a line segment passing through the center of the clotting surface. A longer time evolution of the clot during the initial $600 s$ is visualized in the contour map in Figure 9. Resulting flow deceleration is depicted in Figure 10. 


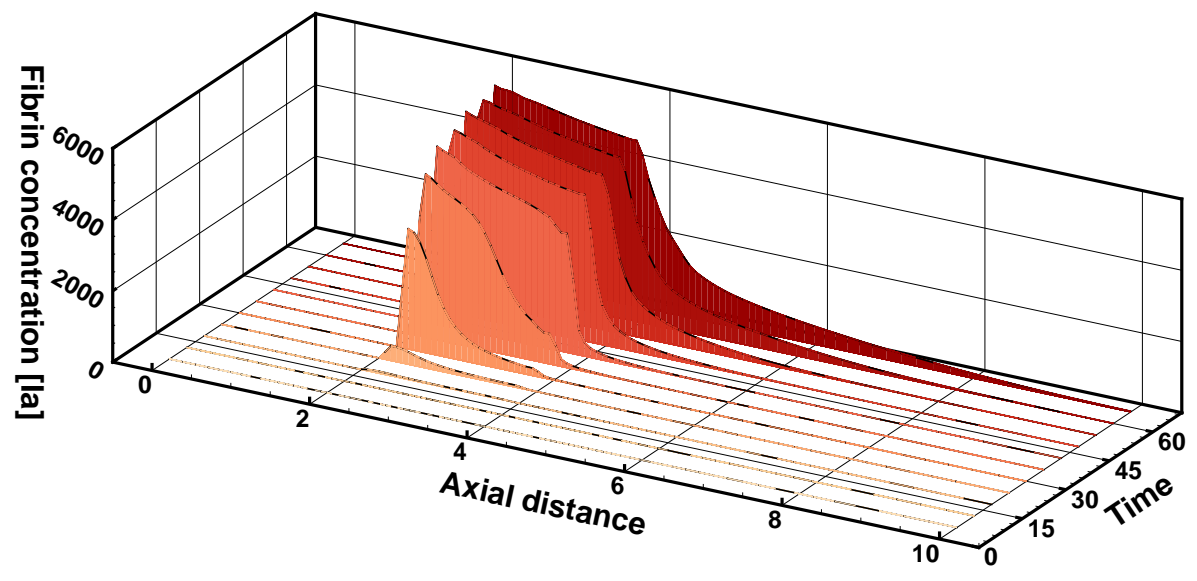

FigURE 8. Fibrin concentration evolution along an axial surface line during the initial 60 seconds.

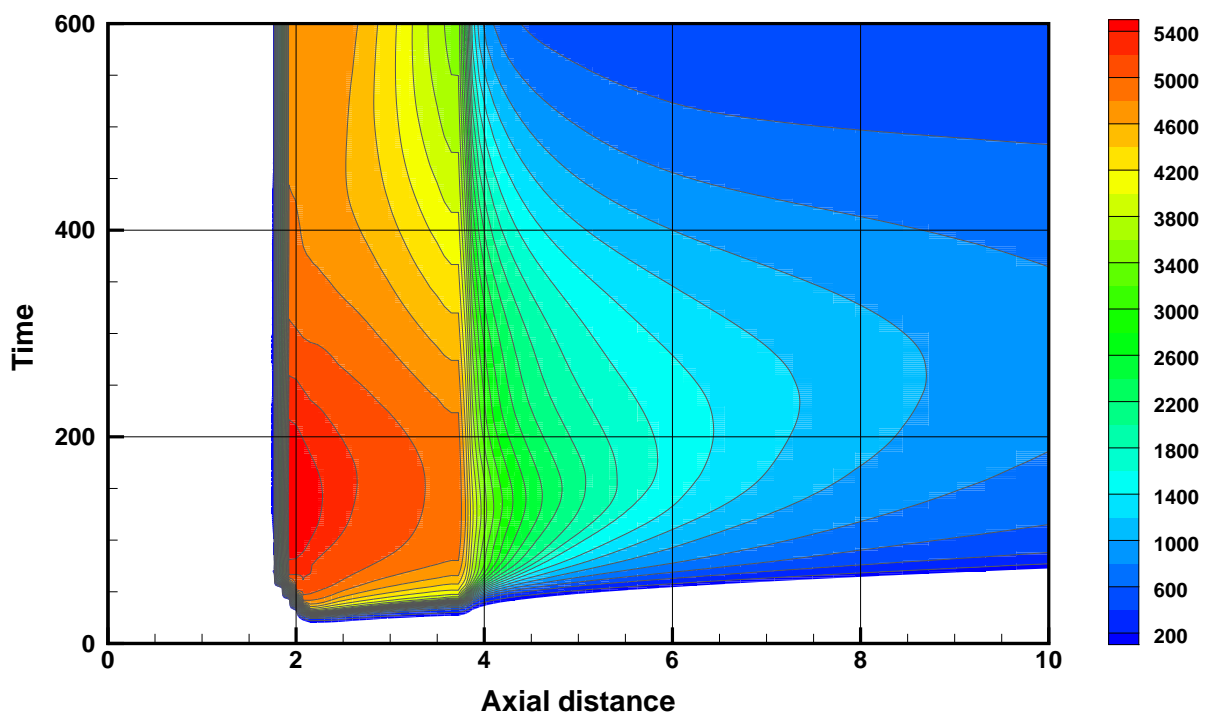

FiguRE 9. Fibrin concentration $[I a]$ along the axial surface line.

\section{Conclusions}

The numerical study presented in this paper has demonstrated the successful implementation of the coupled blood flow and the biochemistry models. The blood flow model is now more complex in comparison with the one presented in our previous work for blood coagulation presented in [10]. The viscoelastic 


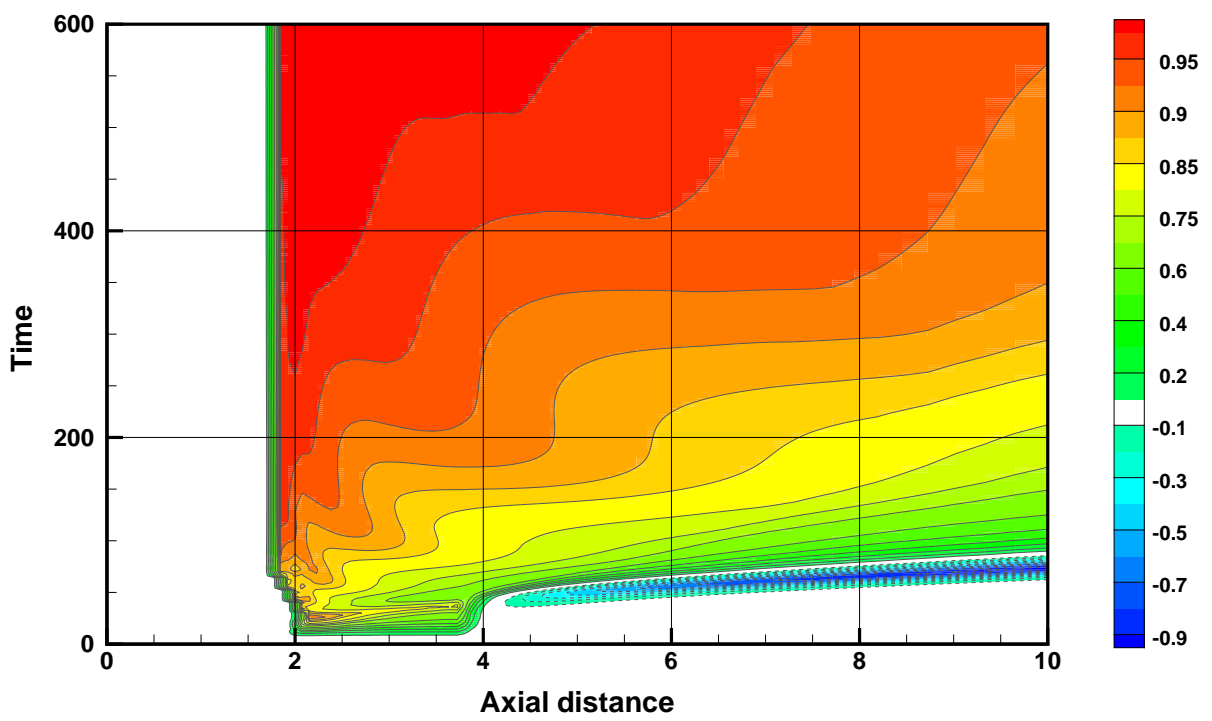

Figure 10. Flow velocity reduction $\delta$ in the near-wall layer along the axial direction.

extension of the model should allow to extend the range of applicability of the model to critical flow regimes. However, the use of this non-linear viscoelastic model extension results in an important increase of the computational costs. The original, generalized Newtonian model with shear-thinning viscosity, contains $4+23=27$ PDEs to solve in 3D. The new model has 6 more equations for the components of the viscoelastic stress tensor. This means that we solved now $4+6+23=33$ equations for the coupled model accounting for the blood rheology and the biochemical processes.

Future research will focus on performance and robustness improvements of the model and numerical solvers. The stability issues raised in [31] should also be addressed in the context of the new modifications of the model. Both of these topics will be important in future applications of the model requiring long time simulations of the clot growth evolution.

Acknowledgements. The financial support of the present work was partly provided by the Czech Science Foundation under the Grant No. P201/11/1304 by and the Portuguese Science Foundation under the Project EXCL/MAT-NAN/0114/2012.

\section{References}

[1] M. Anand, K. Rajagopal, K.R. Rajagopal. A model incorporating some of the mechanical and biochemical factors underlying clot formation and dissolution in flowing blood. J. of Theoretical Medicine, 5 (2003), no. 3-4, $183-218$.

[2] M. Anand, K. Rajagopal, K.R. Rajagopal. A model for the formation and lysis of blood clots. Pathophysiology Haemostasis Thrombosis, 34 (2005), 109-120.

[3] M. Anand, K. Rajagopal, K.R. Rajagopal. A model for the formation, growth, and lysis of clots in quiescent plasma. A comparison between the effects of antithrombin III deficiency and protein $C$ deficiency. J. of Theoretical Biology, 253 (2008), 725-738.

[4] M. Anand, K.R. Rajagopal. A shear-thinning viscoelastic fluid model for describing the flow of blood. Int. J. of Cardiovascular Medicine and Science, 4 (2004), no. 2, 59-68.

[5] M. Anand, K.R. Rajagopal. A mathematical model to describe the change in the constitutive character of blood due to platelet activation. C. R. Méchanique, 330 (2002), 557-562.

[6] F.I. Ataullakhanov, V.I. Zarnitsina, A.V. Pokhilko, A.I. Lobanov, O.L. Morozova. A spatio-temporal dynamics of blood coagulation and pattern formation. A theoretical approach. International Journal of Bifurcation and Chaos, 12 (2002), no. 9, 1985-2002. 
[7] T. Bodnár. On the use of non-linear TVD filters in finite-volume simulations. In: Algoritmy 2012, Proceedings of Contributed Papers and Posters, Slovak University of Technology, Faculty of Civil Engineering, Bratislava (2012), 190-199.

[8] T. Bodnár, J. Př́íhoda. Numerical simulation of turbulent free-surface flow in curved channel. Journal of Flow, Turbulence and Combustion, 76 (2006), no. 4, 429-442.

[9] T. Bodnár, K.R. Rajagopal, A. Sequeira. Simulation of the three-dimensional flow of blood using a shear-thinning viscoelastic fluid model. Mathematical Modelling of Natural Phenomena, 6 (2011), no. 5, 1-24.

[10] T. Bodnár, A. Sequeira. Numerical simulation of the coagulation dynamics of blood. Computational and Mathematical Methods in Medicine, 9 (2008), no. 2, 83-104.

[11] T. Bodnár, A. Sequeira. Numerical Study of the Significance of the Non-Newtonian Nature of Blood in Steady Flow Through a Stenosed Vessel. In: Advances in Mathematical Fluid Mechanics. (edited by R. Rannacher \& A. Sequeira), 83-104, Springer Verlag (2010).

[12] T. Bodnár, A. Sequeira, L. Pirkl. Numerical Simulations of Blood Flow in a Stenosed Vessel under Different Flow Rates using a Generalized Oldroyd - B Model. In: Numerical Analysis and Applied Mathematics, Vols 1 and 2. Melville, New York: American Institute of Physics, vol. 2 (2009), 645-648.

[13] T. Bodnár, A. Sequeira, M. Prosi. On the Shear-Thinning and Viscoelastic Effects of Blood Flow under Various Flow Rates. Applied Mathematics and Computation, 217 (2011), 5055-5067.

[14] S. Butenas, K. G. Mann. Blood coagulation. Biochemistry (Moscow), 67 (2002), no. 1, 3-12. Translated from Biokhimiya, 67 (2002), no. 1, 5-15.

[15] B. Engquist, P. Lötstedt, B. Sjögreen. Nonlinear filters for efficient shock computation. Mathematics of Computation, 52 (1989), no. 186, 509-537.

[16] A. Fasano, R.F. Santos, A. Sequeira. Blood coagulation: a puzzle for biologists, a maze for mathematicians. In D. Ambrosi, A. Quarteroni, and G. Rozza (Eds.) Modeling of Physiological Flows. vol. 5, Modeling, Simulation \& Applications, ch. 3 (2012) 41-75, Springer.

[17] G.P. Galdi, R. Rannacher, A. M. Robertson, S. Turek (Eds.) Hemodynamical Flows: Modeling, Analysis and Simulations. (Oberwolfach Seminars), (2008) Birkhäuser Verlag.

[18] A.M. Gambaruto, J. Janela, A. Moura, A. Sequeira. Sensitivity of hemodynamics in a patient specific cerebral aneurysm to vascular geometry and blood rheology. Mathematical Biosciences and Engineering, 8 (2011), no. 2, 409423.

[19] A. Jameson. Time dependent calculations using multigrid, with applications to unsteady flows past airfoils and wings. In AIAA 10th Computational Fluid Dynamics Conference, Honolulu (1991). AIAA Paper 91-1596.

[20] A. Jameson, W. Schmidt, E. Turkel. Numerical solutions of the Euler equations by finite volume methods using Runge-Kutta time-stepping scheme. In: AIAA 14th Fluid and Plasma Dynamics Conference, Palo Alto (1981), AIAA paper $81-1259$.

[21] J. Janela, A. Moura, A. Sequeira. Absorbing boundary conditions for a $3 D$ non- Newtonian fluid-structure interaction model for blood flow in arteries. International Journal of Engineering Science, 48 (2010), no. 11, 1332-1349.

[22] R. Keslerová. Numerical study of effect of stress tensor for viscous and viscoelastic fluids flow. In: A. Cangiani, R.L. Davidchack, E. Georgoulis, A.N. Gorban J. Levesley, and M.V. Tretyakov (Eds.) Numerical Mathematics and Advanced Applications (2013), 529- 538. Springer. Proceedings of ENUMATH 2011, the 9th European Conference on Numerical Mathematics and Advanced Applications, Leicester, September 2011.

[23] R. Keslerová, K. Kozel. Numerical solution of laminar incompressible generalized newtonian fluids flow. Applied Mathematics and Computation, 217 (2011), no. 11, 5125-5133.

[24] A.L. Kuharsky, A.L. Fogelson. Surface-mediated control of blood coagulation: the role of binding site densities and platelet deposition. Biophysical Journal, 80 (2001), 1050-1074.

[25] K.G. Mann, K. Brummel-Ziedins, T. Orfeo, S. Butenas. Models of blood coagulation. Blood Cells, Molecules, and Diseases, 36 (2006), 108-117.

[26] L. Pirkl, T. Bodnár, K. Tuma. Viscoelastic fluid flows at moderate Weissenberg numbers using Oldroyd type model. In: AIP Conference Proceedings, vol. 1389 (2011), 102-105, American Institute of Physics.

[27] K.R. Rajagopal, A.R. Srinivasa. A thermodynamic frame work for rate type fluid models. Journal of Non-Newtonian Fluid Mechanics, 80 (2000), 207-227.

[28] P. Riha, X. Wang, R. Liao, J.F. Stoltz. Elasticity and fracture strain of blood clots. Clinical Hemorheology and Microcirculation, 21 (1999), no. 1, 45-49.

[29] A.M. Robertson, A. Sequeira, M. Kameneva. Hemorheology. In: Hemodynamical Flows: Modelling, Analysis and Simulation, vol 37, (2008), 63-120, Birkhäuser .

[30] A.M. Robertson, A. Sequeira, R.G. Owens. Rheological models for blood. In:Cardiovascular Mathematics. Modeling and Simulation of the Circulatory System (MS\&A) (2009), 211-241, Springer-Verlag .

[31] A. Sequeira, R.F. Santos, T. Bodnár. Blood coagulation dynamics: Mathematical modeling and stability results. Mathematical Biosciences and Engineering, 8 (2011), no. 2, 425-443.

[32] W. Shyy, M.-H. Chen, R. Mittal, H.S. Udaykumar. On the suppression of numerical oscillations using a non-linear filter. Journal of Computational Physics, 102 (1992), 49-62.

[33] V.I. Zarnitsina, A.V. Pokhilko, F. I. Ataullakhanov. A mathematical model for the spatio-temporal dynamics of intrinsic pathway of blood coagulation - I. The model description. Thrombosis Research, 84 (1996), no. 4, $225-236$. 